\title{
A Novel RTS/CTS based Relative Time Synchronization Technique for RFID based Wireless Sensor Network
}

\author{
Gursimran Kaur $^{\mathrm{a}, *}$, Geetanjali Babbar ${ }^{\mathrm{b}}$ \\ ${ }^{a}$ M.Tech.,Department of Computer Science \& Engg.,Chandigarh Engineering College, Landran(140307),India \\ ${ }^{b}$ Assistant Professor,Department of Computer Science \& Engg., Chandigarh Engineering College, \\ Landran(140307),India
}

\begin{abstract}
Wireless Sensor Network (WSN) is a self-configuring and highly flexible distributed network used for real time monitoring and is comprised of a number of wireless, minute, battery-operated independent sensor nodes connected to a common sink. Due to energy considerations, the network is generally divided into clusters and the entire communication is carried out through the cluster heads. The clock mismatch between various nodes and clusters often leads to collisions, delay and data loss. In order to overcome this problem, a novel RTS/CTS based Relative Time Synchronization Protocol is being proposed for Radio-Frequency Identification based WSN. It achieves better performance due to its energy efficiency and lower service messages. Simulation results show a substantial improvement in the net throughput and reliability of the network when compared with the GPS-based Synchronization technique.
\end{abstract}

Index Terms: Wireless Sensor Network, Radio Frequency Identification, Clustering, Cluster Head, Relative Time Synchronization.

(C) 2015 Published by MECS Publisher. Selection and/or peer review under responsibility of the Research Association of Modern Education and Computer Science

\section{Introduction}

As a result of path-breaking developments in the fields of electronic sensors and wireless communication technology, Wireless Sensor Networks are increasingly becoming useful in wide ranging fields like military surveillance, health monitoring, disaster management, etc. [1,2]. The Wireless Sensor Nodes act as the link between the physical environment and the computing world i.e. the real and virtual world. Generally, a WSN consists of many minute sensor nodes distributed over a large area with a common powerful sink node which acts as a collecting centre for the network data. Data collected from all nodes is processed and analysed at the sink to get useful information. These customised sensors are specifically designed to carry out deep observation

* Corresponding Author: Gursimran Kaur. Tel.: +91-9417917821

E-mail address: gursimrankaur91@gmail.com 
and monitoring of the real world phenomenon through a set of pre-defined parameters. Based on the application requirement and deployment environment, which can be hostile, dangerous or even inaccessible; the sensors to be used may be either embedded or mobile.

Each sensor senses and collects data, processes it into the receiver-defined format and finally propagates it wirelessly to the common network sink. To carry out this entire operation quite frequently and efficiently, the sensors need to configure themselves into an integrated communication network, as the stand-alone information from each sensor has to be pieced together with such information from all other sensors in order to assemble a broader picture of the environment than what each sensor individually senses. Alternatively, [3] terms this process as data fusion, whereby data from each sensor is agglomerated to form a single meaningful result.

RFID (Radio Frequency Identification) is an automatic identification technique which uses a network of tags and readers to accumulate access and propagate data over the network through electromagnetic communication. When used with Wireless Sensor Network, it helps to make the network more target oriented with better message delivery accuracy. In order to further increase the network efficiency and throughput, the technique of clustering is used whereby the network is divided into multiple clusters based on their location and relative energy levels to decrease the collisions and traffic in the network.
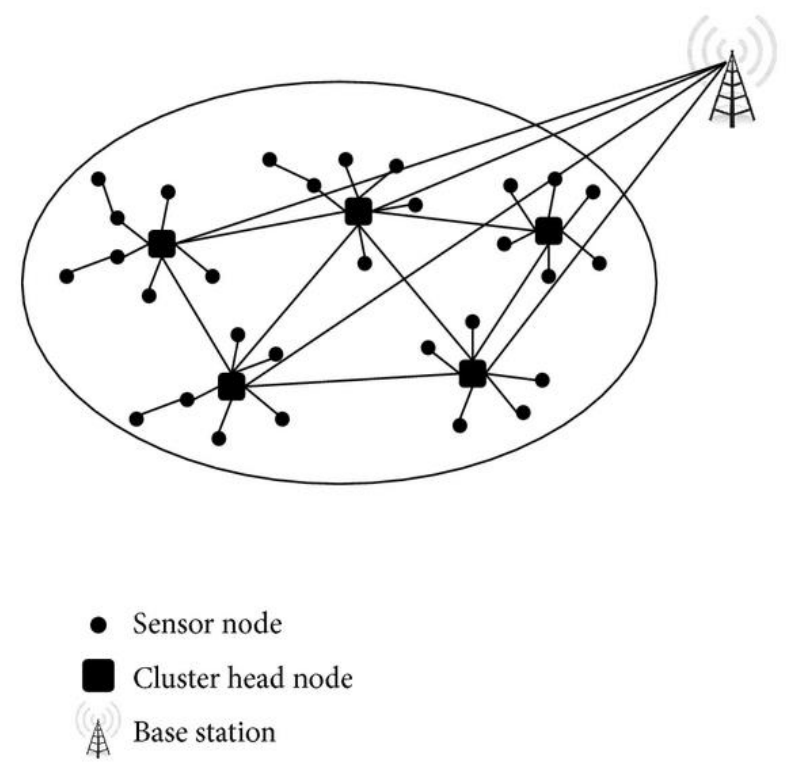

Fig.1. Wireless Sensor Network

Wireless Sensor Networks generally require accurate time synchronization so as to support a variety of operations like packet time-stamping, in-network signal processing, cooperative communication and energy efficient duty cycling of sensor nodes. There are various forms of time synchronization that can be established in the network; such as Absolute Time Synchronization, Relative Time Synchronization, and Local Time Synchronization etc. The time synchronization technique proposed in this paper uses RTS/CTS feed to achieve Relative Time Synchronization in RFID-based clustered WSN.

The rest of this paper is organized as follows. Section II describes the RFID technology in detail while Section III explains clustering in WSNs. Section IV explores the related works and Section V contains the detailed formulation of the problem. The proposed technique has been explained in detail in Section VI while its performance has been analyzed in Section VII. Section VIII concludes the present work. 


\section{Radio Frequency Identification (RFID) based WSN}

RFID is a wireless automated identification technology that has the capability to accumulate and recover data through electromagnetic communication using radio frequency readable integrated circuit. This technology is termed under the group Automatic Identification (Auto ID), such as bar code, magnetic stripes, biometrics (voice, finger printing and retina scanning), smart cards, voice recognition, optical character recognition etc. But RFID system not only enables the feature of unique identification for tracking objects but also overcomes the challenges of the above mentioned identification system. [4]

The RFID technology stores information for a long time. It improves the overall quality of the communication link by using customized radio-frequencies for each node. [5] The system using RFID should be self-configuring.

RFID systems consist of two main components:

a) Tags

b) Readers.

Each RFID tag has a unique identification number (ID) and an associated memory that stores tag data such as the manufacturer, product type etc. It also has values of physical parameters like temperature, pressure, moisture etc. Reader reads and/or writes data to the tags by using the wireless communication channel. Generally, the RFID tags are attached to the objects which are required to be tracked or identified. The values of neighbourhood tags, together with the backend database helps to map the location, movement and relative positioning of the said object [6].

The integration of RFID technology with the Mobile Ad-hoc Networks or Wireless Sensor Networks opens up immense possibilities in both the fields. The collective use of flexibility and mobility of WSNs and the accuracy of the RFID results in manifold improvement in the various performance parameters of the resultant network [7, 8]. The RFID technology is seamlessly embedded in Wireless Sensor Nodes. The active wireless nodes have tags attached to them whereas the passive nodes act as readers for the network. The RFID-based WSN is very effective to carry out error free target based packet delivery in the network. While using the dynamic routing algorithms in WSN, the RFID based nodes provide an efficient way of node identification by using their individual radio IDs.

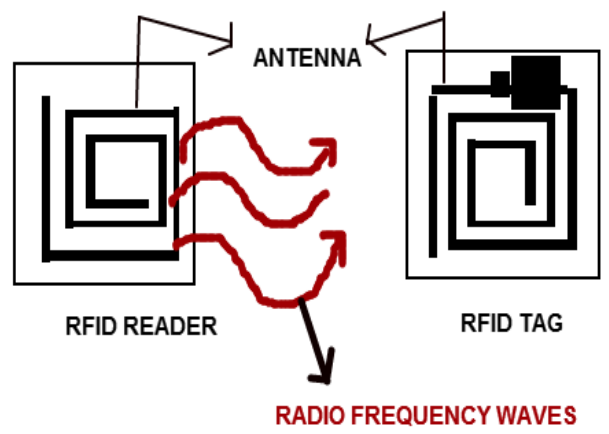

Fig.2. Components of RFID 


\section{Clustering in WSN}

As the wireless sensor network is a resource constraint network, every effort has to be made to bring as much optimization in resource use as possible. In order to save resources and energy, data must be aggregated, and thus, avoiding huge amounts of traffic in the network. The aim of data aggregation is to eliminate redundant data transmission and hence, enhance the life time of the nodes in a wireless sensor network. Data aggregation process has to be done with the help of effective clustering schemes.[9] The grouping of WSN nodes into either homogeneous or heterogeneous clusters is a widely used strategy for obtaining energy efficient and high performing sustainable networks. The use of clusters makes use of some common inter-cluster paths and thus, reduces the net distanced to be traversed by each data packet. Resultantly, significant gains are made in terms of energy conservation in the network. [10]

From time to time, the cluster heads collect data from the cluster nodes and send the data to the common sink [11]. Various commonly used clustering algorithms are LEACH, EEHC, HEED [12], BP, VSA etc. [13] The $\mathrm{CHs}$, thus selected, minimize the overall transmission cost and at the same time balance the power among the nodes, for a longer network lifetime. So, clustering is an important strategy used in WSNs for achieving energy efficiency.

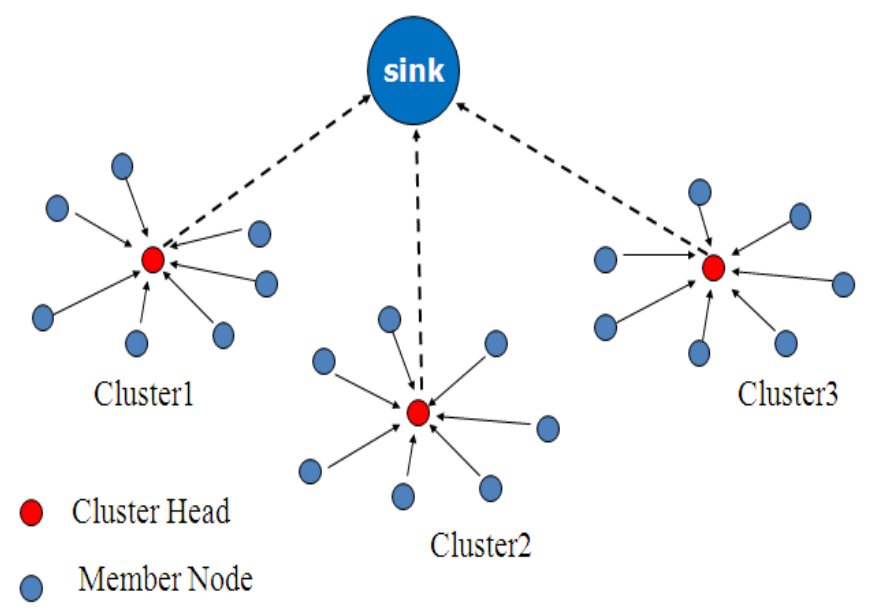

Fig.3. Clustering in WSN

\section{Related Work}

- Chae-Seok Lee et al. [14] purposed Reservation Aloha for No Overhearing that is used to inform the tag of its effective communication for eliminating overhearing problem. The amount of energy consumed due to overhearing is many times larger than the energy consumed for effective communication. To eliminate this problem, author purposes an algorithm (RANO). A tag has advance information about the time and duration of the communication as it maintains active mode during the sleeping mode of others in the transmission period.

- Jian-qi et al. [15], proposed improved Clustering Routing Algorithm which gives priority to energy efficiency. First, it generates a cluster head in each cluster by random competition in the nodes having advantage in energy; next it determines the internal structure of the clusters by dynamically calculating the tightness coefficient of each cluster; after that, it optimizes the transmission path between cluster heads through improved Multi-Objective Particle Swarm Algorithm. 
- Nicolas Gouvy et al. [16], proposed PAMAL (PATH MERGING ALGORITHM), a new geographies routing algorithm for mobile nodes. The proposed algorithm is the first routing protocol which uses paths crossings to adapt the network topology to simultaneously reduce the network traffic and maximize energy efficiency. The protocol makes the intersection to move away from the destination, getting closer to the sources, allowing higher data aggregation and thus, saving energy.

- Degan Zhang et al. [17], proposed a method Forward Aware Factor (FAF-EBRM) which is used for the selection of the next hop node according to the forward energy density and link weight .The FAF-EBRM is comparable to LEACH and EEUC. The proposed method balances the energy reduction, function lifetime and provides good quality of service. It also reduces the probability of successive node breakdown.

\section{Problem Formulation}

In a Wireless Sensor Network, the sensor recordings and timestamps are passed along so that the fusion of such information from various sources will agglomerate to form a global result at the sink node. Now in such a case, there may be a time mismatch among various sensors which can lead to information leakage or ambiguity. The differences amongst their individual clocks can often lead to packet loss on account of collisions, transmission redundancy and delay.

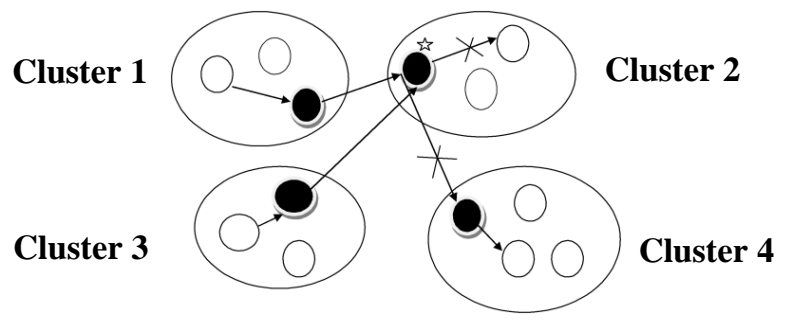

Fig.4. A Network with Asynchronous Nodes and Packet Collision

Various algorithms and protocols have been proposed for synchronizing the clocks within the network, such as Network Time Protocol (NTP), Precision Time Protocol (PTP), Reference Broadcast Synchronization (RBS), and GPS-based Synchronization etc. But all these protocols have their individual drawbacks ranging from low accuracy, high cost, and energy inefficiency to high complexity. In this manner, being a limited-energy system, the efficiency, lifetime and robustness of the WSN is severely compromised. The present work intends to counter this problem in the Wireless Sensor Networks by proposing a new RTS/CTS based algorithm.

\section{Proposed Algorithm}

Liskov [18] states that clock synchronization improves the performance of distributed algorithms by replacing communication with local computation. Any clock synchronization protocol should fulfil the following requirements:

1. It should cope with unreliable network transmission and unbounded message latencies.

2. During mutual synchronizing, each node must be able to estimate the local time on the other node's clock.

3. Time must never run backward. Clocks should be gradually incremented until the required correction is achieved. 
4. Synchronization overhead must not degrade system performance.

\subsection{Two-Step Synchronization}

In order to obtain an energy-efficient Time Synchronization solution to the above said problem in RFIDbased WSN, the RTS/CTS based Relative Time Synchronization technique is used. For this purpose, a two step methodology has been proposed to synchronize the whole network. In the first step, only Cluster Heads are synchronized and in the second step, sensor nodes in the cluster will be synchronized.

\section{Step I:}

Firstly, the WSN is deployed and divided into clusters. Then the respective Cluster Heads (CHs) are elected and every $\mathrm{CH}$ informs the SINK about its selection and responsiveness. Now the SINK would send an RTS (Request to Send) message which contains the NONCE feed to each $\mathrm{CH}$. The NONCE feed is a cryptographic number to be used only once, generated for a specific purpose and contains the clock time of the SINK. When the CHs receive the RTS packets, they will represent their respective clocks. The head nodes then send CTS (Clear to Send) packets to SINK. After sending CTS packets to SINK, cluster heads would adjust their clocks according to time of the NONCE feed received from the SINK. Now, all the CHs are relatively synchronized with the common SINK.

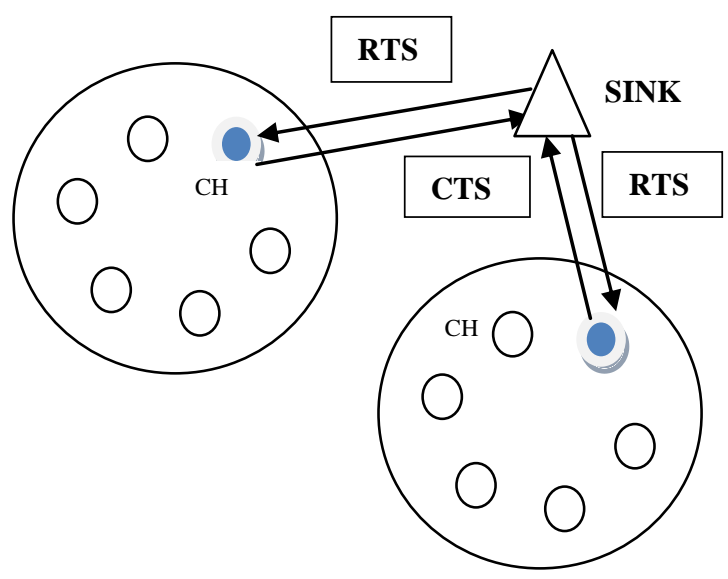

Fig.5. Synchronization of Cluster Heads

Step II:

Now all the CHs will send a PING message containing a time feed to all its cluster members. The member nodes would adjust their clocks according to the feed and revert back to their respective Cluster Heads with a PING message signifying successful time synchronization with the $\mathrm{CH}$. 


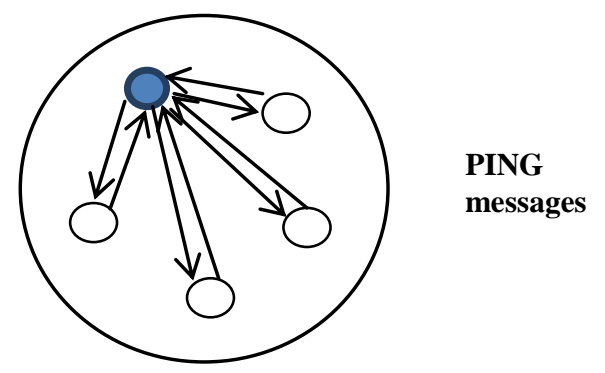

Fig.6. Synchronization of Member Nodes

In this way, the whole network gets synchronized and slotted ALOHA works perfectly in the network with negligible packet losses.

\subsection{Algorithm}

1. Base Station:

2. broadcast (Sync_start, level=0)

3. If receive (Sync_req) then

4. $\quad$ send (Sync_ack, T1, T2, T3)

5. End if

6. Neighbour cluster nodes:

7. receive (Sync_start, level)

8. if $($ level $=$ null $)$ then

9. level++;

10. wait a short random time ;

11. send (Sync_req, level, T1) ;

12. ifreceive( Sync_ack)then

13. $\operatorname{record}(\mathrm{T} 1, \mathrm{~T} 2, \mathrm{~T} 3, \mathrm{~T} 4)$;

14. $\mathrm{d}=((\mathrm{T} 2-\mathrm{T} 1)-(\mathrm{T} 4-\mathrm{T} 3)) / 2$;

15. calculate (d);

16. Sync(d);

17. End if

18. End if

19. Broadcast $($ Sync_start,node $=0)$

20. if node(receiver Sync)then

21. Clusterhead send(ping)

22. if(Node receive Ping)then

23. Send(Ack)

24. Wait for random time

25. Node record(d and d1)

26. if $(\mathrm{d} 1==\mathrm{d})$ then

27. Node adjust its clock to $\mathrm{d}$

28. else

29. Reply with OK message

$30 . \quad$ End if

31. End if 


\section{End if}

\section{Performance Evaluation}

\subsection{Simulated Implementation}

In order to evaluate the performance of the proposed algorithm, it has been simulated in the Network Simulator 2 (ns2). The Network Simulator is a discrete event packet level simulator which consists of tools that simulate behaviour of networks such as creating network topologies, traffic models, load patterns etc.

Table 1. Simulation Parameters

\begin{tabular}{ll}
\hline \multicolumn{1}{c}{ Parameter } & \multicolumn{1}{c}{ Description } \\
\hline Channel & Wireless \\
\hline Number of Mobile Nodes & Omni \\
\hline Link layer type & LL \\
\hline Topology & Random \\
\hline Mobility & High (pause time 0) \\
\hline Mobility Model & Random Walk \\
\hline Interface queue type & DropTail/PriQueue \\
\hline MAC type & Mac/802_11 \\
\hline Network Interface Type & Phy/WirelessPhy \\
\hline Radio-Propagation Model & TwoRayGround \\
\hline Routing Protocol & AODV \\
\hline
\end{tabular}

The use of random topology, random walk mobility model, and pause time of zero makes the network highly dynamic. For individual identification of the nodes over the network, the RFID technology is used. The routing protocol used is the AODV (Ad-hoc On Demand Distance Vector) protocol which supports both unicast and multicast routing over the mobile ad-hoc networks. The TDMA based MAC protocol called LEACH (Low Energy Adaptive Clustering Hierarchy) protocol is used for carrying out the elections of the Cluster Heads. The performance of the RTS/CTS based Time Synchronization Protocol has been compared with the GPS-based Time Synchronization Protocol.

\subsection{Efficiency Analysis}

The Network parameters under consideration for performance evaluation are as under: 
- Energy used by the network per unit time (in joule per second)

- Magnitude of Packet loss in the network per unit time.

- The network throughput (in terms of number of successful transmissions in the network per unit time)

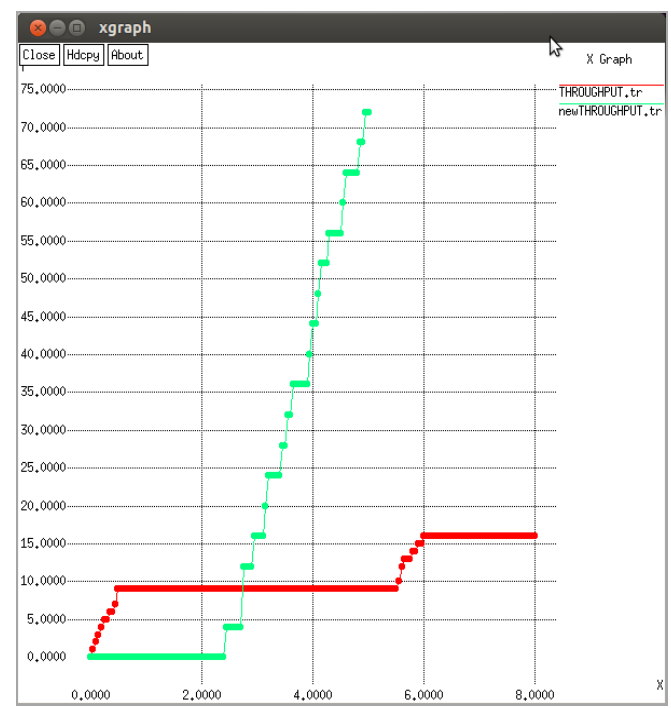

Fig.7. Network Throughput per Unit Time

Figure 7 shows the net throughput of the network in the terms of number of packet successfully transmitted per unit time. The throughput while using the proposed methodology is as high as 73,000 successful data transmissions per unit time; whereas the throughput value of the GPS-based network is far less at about 16,000 successful transmissions per unit time.

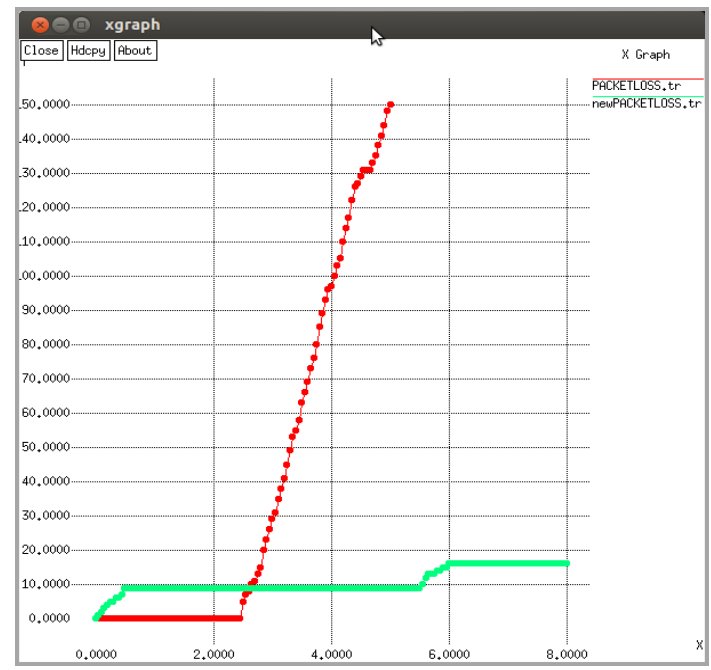

Fig.8. Number of Packets Lost Per Unit Time

Figure 8 shows that the number of packets lost during in the network per unit time due to packet collisions 
has been brought from about 50,000 per unit time in the other protocol to about 18,000 per unit time in the proposed methodology.

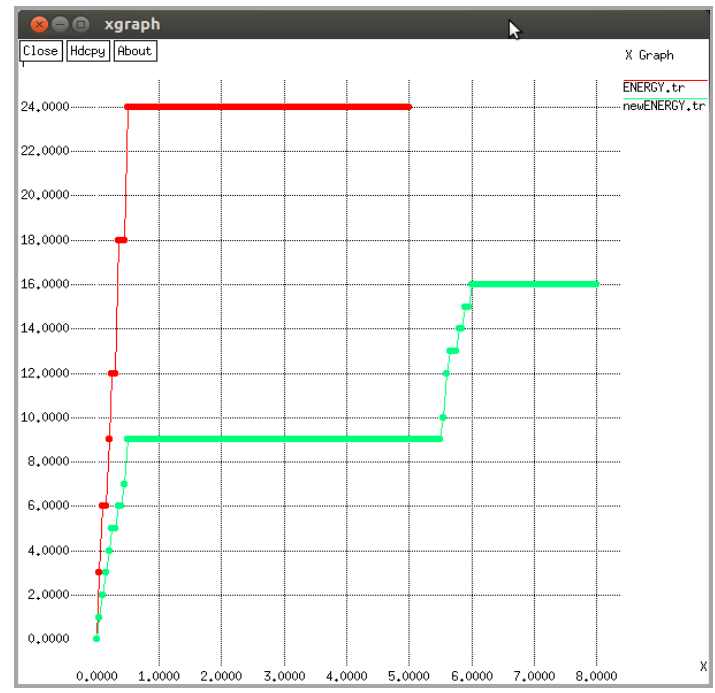

Fig.9. Energy Consumed In Joules per Unit Time

The energy analysis of the two protocols shows that although the absolute time synchronization has various inherent advantages but this approach leads to massive and sudden drain in the network energy due to the use of GPS technology (shown in red). On the other hand, the RTS/CTS based strategy (shown in green) shows a two-step energy usage which is still less than the former technique.

Here, the $\mathrm{X}$-axis represents time per second and the $\mathrm{Y}$-axis shows the energy in joule. The lower energy utility of the RTS/CTS based technique provides the following advantages to the network:

- It increases the lifetime of the cluster heads involved in the transmission.

- The increase in the lifetime of cluster heads eliminates the need for holding frequent elections in the clusters.

- The decrease in the rate of change of cluster heads reduces the overall packet loss in the network.

- The increase in the lifetime of the whole network raises the usability, efficiency, authenticity and sustainability of the network.

\section{Conclusion}

As increasing demand in use and promising application of sensor network are emerging, precise and secure clock measurement algorithms are vital for error free clock time measurements. Traditional clock synchronization protocols for wired networks cannot be used because wireless sensor network protocols require the ability to adapt dynamically, the ability to handle sensor mobility, and scalability. The sensors themselves are heavily resource-constrained because of limited battery power. Furthermore, they need to operate in highly losing and unreliable environments. As a result, several clock synchronization protocols for wireless sensor networks have been designed in the recent past. For achieving Relative Time Synchronization in Radio Frequency Identification (RFID) based Wireless Sensor Network, a novel RTS/CTS based protocol has been proposed in the present work. It uses a two-step strategy to synchronize the whole clustered WSN with the 
common SINK or Base Station. In the first step, all the Cluster Heads synchronize their clocks with the SINK by using the NONCE feed supplied by the sink in RTS message. The successful synchronization is acknowledged by the Cluster Head in the CTS message. In the second step, all the Cluster Heads send their time feed to their respective member nodes through a Ping message. After successful synchronization, the member nodes revert back with the Ping message. Simulation results have shown that, when compared with the GPS-based synchronization technique, the proposed technique shows a substantial improvement in the net throughput and reliability of the network.

\section{References}

[1] Akyildiz I.F., W. Su, Sankarasubramaniam Y., Cayirci E., "Wireless sensor networks: A survey", Computer. Netw.2002, 38, 393-422.

[2] Al-Karaki J.N.; Kamal A.E., "Routing techniques in Wireless Sensor Networks: A survey", IEEE Wirel. Commun. 2004, 6-28.

[3] Sundararaman Bharath, Buy Ugo, and Kshemkalyani A.D., "Clock Synchronization for Wireless Sensor Networks: A Survey", University of Illinois, pp. 1-49, 2005.

[4] Ahmes J. et. at., "RFID-WSN Integrated Architecture for Energy and Delay-Aware Routing", SpringerBriefs in applied Sciences and Technology, 978-981-287-414-6_1.

[5] Chiesa M. et al, "RFID: A Week Long Survey on theTechnology and Its Potential," Technical Report on Hamessing Technology Project, March 2002.

[6] Vaidya N., and Das S.R., "RFID-Based Networks - Exploiting Diversity and Redundancy," Technical Report: June 2006.

[7] Bashir Ali Kashif, Park Myong-Soon, et.al, "In-network RFID Data Filtering Scheme in RFID-WSN for RFID Applications", National Fusion Research center, Korea.

[8] Najera Pablo, Roman Rodrigo and Lopez Javier, "Secure Architecture for the Integration of RFID and Sensors in Personal Networks", University of Malaga, Spain.2012.

[9] Maraiya Kiran, Kant Kamal and Gupta Nitin, "Efficient Cluster Head Selection Scheme for DataAggregation in Wireless Sensor Network", International Journal of Computer Applications (0975 8887), Volume 23-No.9, June 2011.

[10] Forero.P. A., Alfonso Cano, and Giannakis Georgios B., "Distributed Clustering Using Wireless Sensor Networks", IEEE Journal of selected topics in Signal Processing, Vol. 5, no. 4, august 2011707.

[11] Zhang and Damianos Gavalas,"RFID and Sensor Networks", AU7777 C012, Page 323 2009-6-24.

[12] Yaghmaee Mohammad Hossein and Mahmoud Naghibzadeh, "A Local Cluster Head Election Algorithm inWireless Sensor Networks", 978-1-61284-207-3/11, 2011 IEEE.

[13] Anker Tal, Bickson D., Bracha Hod and Dolev Danny, "Efficient Clustering for Improving Network Performance in Wireless Sensor Networks", The Hebrew University of Jerusalem, Israel.

[14] Kim Dong-Hyun, Kim Jong-Deok and Lee Chae-Seok "An Energy Efficient Active RFID Protocol to Avoid Overhearing Problem", IEEE, vol.14, no.1, pp 15-24, Jan.2014.

[15] Jiang, L., Bing Fang, \& Li. (May, 2013) Energy optimized approach based on clustering routing protocol for wireless sensor networks. CCD Conference. IEEE.

[16] Gouvy, N., Hamouda, E., Mitton, N., \& Zorbas, D. (2013, April). Energy efficient multi-flow routing in mobile Sensor Networks. In Wireless Communications and Networking Conference (WCNC), 2013 IEEE (pp. 1968-1973). IEEE.

[17] Zhang, D., Li, G., Zheng, K., Ming, X., \& Pan, Z. H. (2014). An Energy-Balanced Routing Method Based on Forward-Aware Factor for Wireless Sensor Networks. Industrial Informatics, IEEE Transactions on, 10(1), 766-773.

[18] B. Liskov, "Practical Uses of Synchronized Clocks in Distributed Systems", Proc. Tenth Annual ACM Symposium on Principles of Distributed Computing, pp. 1-9, Aug. 1991. 


\section{Author(s) Profiles}

Gursimran Kaur is pursuing M.Tech from Chandigarh Engineering College, Punjab (India). She received her B.Tech. degree in Computer Science \& Engineering in 2013 from Punjab Technical University. Her areas of interest include wireless communication, ad-hoc sensor networks and distributed computing.

Geetanjali Babbar is an M.Tech. in Computer Science \& Engineering and is presently serving as an Assistant Professor in Chandigarh Engineering College, Punjab (India). She has guided numerous students in their postgraduate research.

How to cite this paper: Gursimran Kaura, Geetanjali Babbar,"A Novel RTS/CTS based Relative Time Synchronization Technique for RFID based Wireless Sensor Network", IJWMT, vol.5, no.5, pp.35-46, 2015.DOI: $10.5815 /$ ijwmt.2015.05.04 